\title{
Breast MRI in the setting of DCIS: quality trials are still needed to determine its value
}

\author{
Habib Rahbar ${ }^{1}$ (D) Judy A. Tjoe ${ }^{2}$ \\ Received: 24 March 2021 / Accepted: 6 May 2021 / Published online: 28 May 2021 \\ (C) European Society of Radiology 2021
}

\section{Key Points}

- Existing evidence suggests that preoperative breast MRI does not improve treatment outcomes for DCIS.

- The quality of the available data used to answer this question is poor.

- Quality randomized controlled trials are needed to definitively assess the benefits and harms of preoperative MRI in the setting of DCIS.

Ductal carcinoma in situ (DCIS) of the breast represents the earliest form of breast cancer diagnosed and is typically detected on screening mammography as asymptomatic calcifications. Nearly every aspect of the DCIS care pathway is controversial, starting from whether it constitutes a true cancer, the pathological criteria by which it is diagnosed and classified, its natural history, and the appropriateness of its generally aggressive standard-of-care management. Despite these controversies, surgery remains the centerpiece of DCIS management in the overwhelming majority of women diagnosed with DCIS, with margin status of paramount importance. Compared to its invasive counterpart, DCIS reexcision rates are relatively high (30-40\%), likely due to lack of palpability, segmental distribution with skip areas that are non-calcified, and more stringent guidelines on adequate margins [1]. Because DCIS diagnosis is associated with the visualization of mammographic calcifications, it was initially

This comment refers to the article available at https://doi.org/10.1007/ s00330-021-07873-2.

Habib Rahbar

hrahbar@uw.edu

Judy A. Tjoe

jatjoe@novanthealth.org

1 Department of Radiology, University of Washington School of Medicine, Seattle Cancer Care Alliance, 1144 Eastlake Ave East Rm LG-200, Seattle, WA 98109, USA

2 Department of Breast Surgery, Novant Health Surgical Institute, 3515 West Market Street, Suite 300, Greensboro, NC 27403, USA postulated that MRI would provide little value for its detection and visualization. This thinking changed after a landmark study by Kuhl et al who demonstrated that MRI provides substantially higher sensitivity than mammography for its detection [2]. Many studies have also demonstrated that MRI likely provides a more accurate estimate of final pathological DCIS span than mammography alone [3]. Furthermore, due to its reliance on suspicious enhancement that serves as a marker of abnormal vascularity, MRI may be particularly useful for identifying higher grade forms of DCIS less likely to represent overdiagnosis.

Despite this promise, the practical benefit of improved DCIS depiction with MRI remains unclear and hotly debated. A metaanalysis of randomized controlled trials and observational studies of MRI and DCIS published in 2015 demonstrated no reduction in re-operation rate with pre-operative MRI [4], though it was acknowledged that many of the included studies predated published guidelines on high-quality breast MRI acquisition and interpretation. In this paper in European Radiology by Canelo-Aybar et al, the European Commission Initiative on Breast Cancer performed an extensive updated systematic review on the impact of MRI in DCIS management, adding both newer randomized controlled trials and single-institution observational studies [5]. The authors provide estimates of the effect of MRI on outcomes and are transparent in their assessment of the quality of evidence using the Grading of Recommendations, Assessment, Development and Evaluations (GRADE) framework [6]. In this important study, they found no clear evidence that pre-operative MRI to evaluate DCIS provides an impact on surgical outcomes or local recurrence but noted that the evidence was of "low to very low certainty." 
Many surgeons and radiologists anecdotally see value in pre-operative breast MRI to visualize enhancement patterns that suggest more extensive DCIS or occult invasive disease. Thus, it may be perplexing for these clinicians that the evidence for MRI to date does not prove this value. Nevertheless, it is equally remarkable that Canelo-Aybar's results highlight a striking lack of quality evidence in this domain. The specific GRADE adjectives used to describe the quality of data indicate that the true effect of MRI on surgical and recurrence outcomes is either "probably markedly different" or "might be markedly different from the estimated effect [6]." Why so much uncertainty despite decades of breast MRI research? The answer partly lies in the fact that there are many challenges when conducting a meta-analysis or systematic review of observational studies regarding the value of breast MRI, a highly sensitive imaging modality with a wide range in performance (depending on technique quality and radiologist training) and utilization rates (based on geography, resources, clinician and patient preferences, among other factors). Furthermore, there is a lack of sufficiently powered quality randomized controlled trials incorporating minimum standards for MRI acquisition and management of findings. Specifically, the two most well-known pre-operative MRI randomized controlled trials included in this systematic review, the MONET and COMICE trials, had very different designs, and neither trial included only patients with DCIS. In the MONET trial, MRI preceded core needle biopsy, which is not a typical pre-operative approach. While MRI was performed after core needle biopsy but preceding surgery in the COMICE trial, the study suffered from a lack of MRI technical and interpretation quality standards, low recruitment at individual sites, a high mastectomy rate performed without confirmation of MRI findings, and an overrepresentation of post-menopausal women.

Although not included in this meta-analysis due to a lack of no-MRI control arm, it is notable that the ECOG-ACRIN Cancer Research Group E4112 trial demonstrated that when quality MRI is incorporated into the management of women with DCIS with clear management guidance, successful WLE rate is very high (96\%) and only $22.1 \%$ who underwent an initial WLE required re-excision(s) [7]. This is paramount from dual perspectives: societies and institutions using reexcision lumpectomy rates as a quality metric place value on low re-excision rates [8], while patients express greater satisfaction when the first surgery to treat DCIS is the final surgery [9]. Furthermore, newer retrospective observational studies and a recent small prospective randomized controlled trial (IRCIS) [10] have also suggested greater promise for reducing re-excisions without increasing mastectomy rates. While these studies were included in Canelo-Aybar et al's report, their effects are likely diluted by the inclusion of many earlier publications that did not carefully control for other factors known to impact MRI and surgical performance. The authors therefore appropriately conclude that there is a need to perform a large randomized controlled trial to definitively assess this important clinical question. Finally, it is important to note that this systematic review was also unable to account for the range in practice as it relates to the selection of adjuvant therapies for DCIS treatment in the setting of pre-operative MRI, the promise of radiomics to assess DCIS prognosis, or the impact of social determinants of health on DCIS outcomes. Given the remarkable number of controversies associated with DCIS and the costs associated with randomized controlled trials, future trials of DCIS and advanced imaging would be well-served to also carefully consider these secondary questions so that we may maximize study value, impact, and translation.

Funding This editorial has received funding from the National Institutes of Health NCI R01CA203883 (Rahbar, PI).

\section{Declarations}

Guarantor The scientific guarantor of this publication is Habib Rahbar, MD.

Conflict of interest The authors of this manuscript declare relationships with the following companies:

GE Healthcare, co-investigator on grant paid to institution unrelated to this work.

Statistics and biometry No complex statistical methods were necessary for this paper.

Informed consent N/A

Ethical approval N/A

Methodology

- Editorial comment

\section{References}

1. Shehata M, Grimm L, Ballantyne N et al (2019) Ductal carcinoma in situ: current concepts in biology, imaging, and treatment. J Breast Imaging 1:166-176

2. Kuhl CK, Schrading S, Bieling HB et al (2007) MRI for diagnosis of pure ductal carcinoma in situ: a prospective observational study. Lancet 370:485-492

3. Bartram A, Gilbert F, Thompson A, Mann GB, Agrawal A (2021) Breast MRI in DCIS size estimation, breast-conserving surgery and oncoplastic breast surgery. Cancer Treat Rev 94:102158

4. Fancellu A, Turner RM, Dixon JM, Pinna A, Cottu P, Houssami N (2015) Meta-analysis of the effect of preoperative breast MRI on the surgical management of ductal carcinoma in situ. Br J Surg 102: 883-893

5. Canelo-Aybar C, Taype-Roldan A, Zafra-Tanaka JH et al (2021) Preoperative breast magnetic resonance imaging in patients with ductal carcinoma in situ: a systematic review for the European Commission Initiative on Breast Cancer (ECIBC). Eur Radiol. https://doi.org/10.1007/s00330-021-07873-2 
6. Balshem H, Helfand M, Schunemann HJ et al (2011) GRADE guidelines: 3. Rating the quality of evidence. J Clin Epidemiol 64: 401-406

7. Lehman CD, Gatsonis C, Romanoff J et al (2019) Association of magnetic resonance imaging and a 12-gene expression assay with breast ductal carcinoma in situ treatment. JAMA Oncol. https://doi. org/10.1001/jamaoncol.2018.6269

8. The American Society of Breast Surgeons Official Statement: Consensus Guideline on Breast Cancer Lumpectomy Margins. Available at https://www.breastsurgeons.org/docs/statements/ Consensus-Guideline-on-Breast-Cancer-Lumpectomy-Margins. pdf Accessed on March 24, 2021
9. Carlos RC, Snyder BS, Lehman CD et al (2017) Decision quality and quality of life after treatment for DCIS: a trial of the ECOGACRIN Cancer Research Group (E4112). Presented at 2017 Radiological Society of North America Annual Meeting, High Impact Clinical Trials Session, Chicago, IL

10. Balleyguier C, Dunant A, Ceugnart L et al (2019) Preoperative breast magnetic resonance imaging in women with local ductal carcinoma in situ to optimize surgical outcomes: results from the randomized phase III trial IRCIS. J Clin Oncol 37:885-892

Publisher's note Springer Nature remains neutral with regard to jurisdictional claims in published maps and institutional affiliations. 